\section{Widespread Cortical Lesions on Diffusion-Weighted Imaging in Acute Portal Systemic Shunt Encephalopathy Caused by Primary Biliary Cirrhosis}

Hepatic encephalopathy (HE) is 1 cause of a disturbance of consciousness, most cases being associated with liver cirrhosis. There are 2 previous reports of diffusion-weighted imaging (DWI) of the brain in acute HE. ${ }^{1,2}$ We now report a rare case of shunt encephalopathy caused by primary biliary cirrhosis (PBC) with widespread cortical lesions on brain DWI.

A 72-year-old woman experienced a disturbance of consciousness for 2 days before coming to our emergency department. At her former hospital, she had been followed for 4 years for pancytopenia of unknown origin. She had been treated for gastric ulcer 5 years ago but without a blood transfusion. She was not dependent on alcohol, and her family history was negative for disturbance of consciousness. Neurologic examination showed mild disturbance of consciousness (Glasgow Coma Scale score 14). Flapping tremor was absent, and there were no other neurologic abnormalities. Dilated superficial abdominal veins were present in her lower abdomen.

Laboratory examinations disclosed thrombocytopenia $(8.8 \times$ $10^{4} / \mu \mathrm{L}$ ) and an elevated alkaline phosphatase level (431 IU/L). Other serum levels for liver enzymes, albumin, vitamins, inflammation response, and prothrombin time were within the normal range. HBsAg

Indicates open access to non-subscribers at www.ajnr.org and anti-hepatitis C virus antibody levels were negative. Her CSF showed mild pleocytosis $\left(8 / \mathrm{mm}^{3}\right)$ without protein elevation, low glucose levels, or cultures positive for bacteria, fungi, and tubercle bacilli. Antigens for herpes simplex, herpes varicella zoster, Epstein-Barr, and cytomegalovirus in her serum and CSF were negative for acutephase infection. No abnormal intensity was seen on DWI of the brain performed at admission. Acyclovir was administered intravenously, but she developed a deep coma with snoring-like breathing on the second hospital day. Intubation and mechanical ventilation were required. Brain DWI on the same day showed widespread highintensity areas at the bilateral frontal, temporal, and parietal cortices (Fig 1A). The same areas had low values on an apparent diffusion coefficient (ADC) map (Fig 1B) with no gadolinium enhancement (Fig 1C). The lesion area looked normal on T1-weighted images and high-intensity T2-weighted images. Laboratory data obtained on the same day detected an elevated blood ammonia level $(299 \mu \mathrm{g} / \mathrm{dL})$.

Abdominal CT with contrast enhancement showed a splenorenal shunt with neither splenomegaly nor liver cirrhosis. Elevated indocyanine green test $(27.3 \%)$ and low Fisher ratio (1:5) values were compatible with portal systemic shunt encephalopathy. Antimitochondrial $\mathrm{M}_{2}$ antibody was positive. The diagnosis was grade IV HE caused by PBC. She was administered branched-chain amino acid-rich fluid intravenously, which improved her consciousness. The respirator was removed on the seventh hospital day. Her blood ammonia level was lowered by repeated doses of lactulose, ursode-
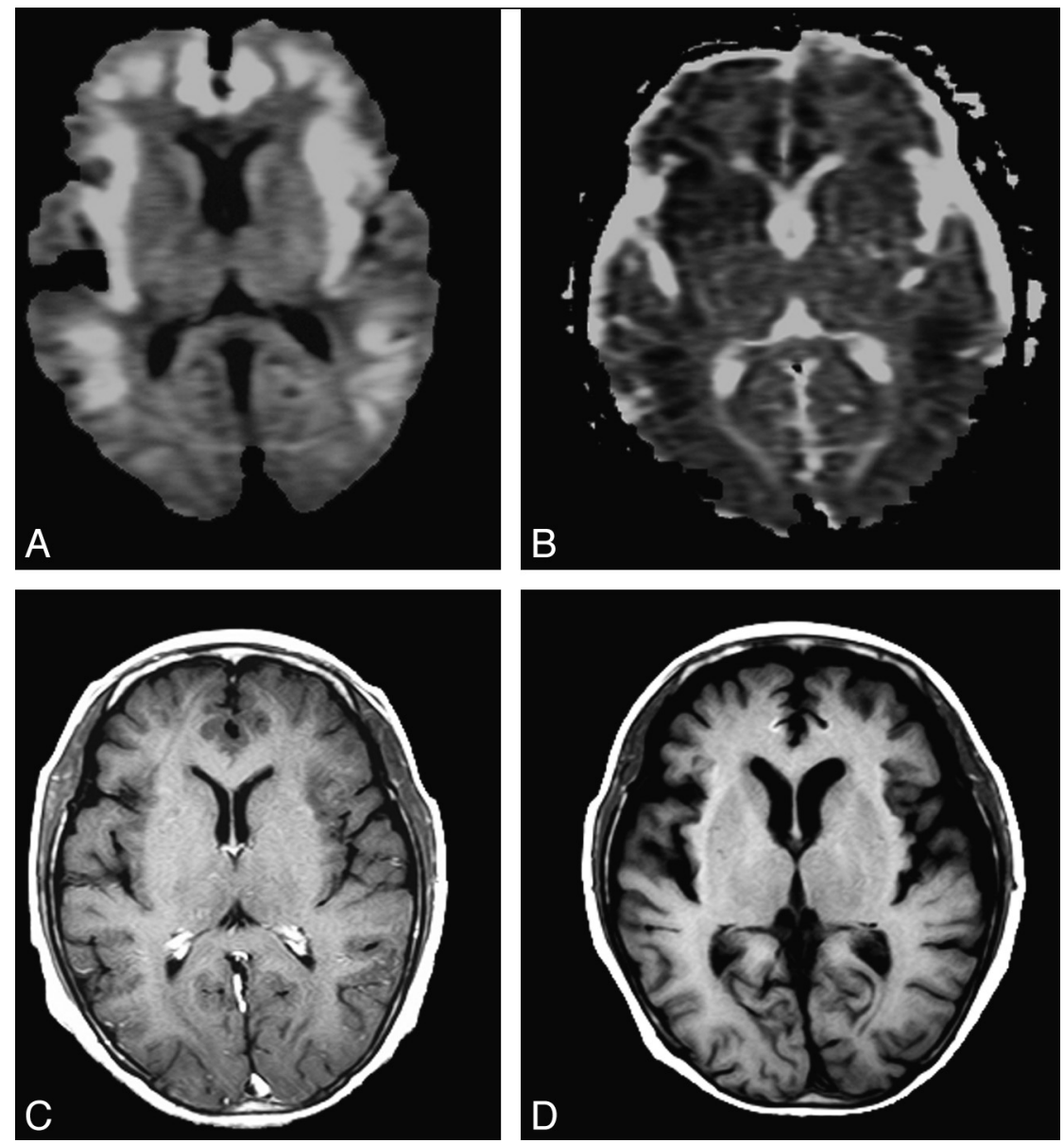

Fig 1. The patient's brain on MR imaging. $A$, On the second hospital day, widespread high-intensity areas are present at the diffuse cortex, sparing the occipital cortex on DWI. $B$ and $C$, The ADC map on the same day shows low values at the cortex $(B)$ and no enhancement on the T1-weighted image with gadolinium $(C)$. $D$, Cortical laminar necrosis is present on the T1-weighted image on the 54th day. 
oxycholic acid, and branched-chain amino acids. Brain MR imaging on the 54th hospital day showed no DWI high-intensity areas. Highintensity areas present in the frontotemporal cortex on T1-weighted images, however, were equivalent to cortical laminar necrosis (Fig $1 D)$. She was discharged on the 55th hospital day without sequelae.

Only 2 cases of DWI abnormality in acute HE have been reported. ${ }^{1,2}$ One was acute $\mathrm{HE}$ in a patient with alcoholic cirrhosis; the other patient with $\mathrm{HE}$ had type $\mathrm{C}$ cirrhosis after transjugular intrahepatic portosystemic shunt placement. These patients showed diffuse cerebral cortical lesions on DWI, whereas the occipital cortex was spared as in our patient. In case 2, low ADC values were documented in the affected area.

Acute HE has an acute phase followed by a chronic one. Pathologically, during the acute phase, it is considered to be brain edema, and in the chronic phase, there is a thin cortex and cortical laminar necrosis. ${ }^{3}$ The proposed cytotoxic edema mechanism in HE is hyperammonia inducing intracerebral accumulation of glutamine, resulting in astrocyte swelling and brain edema. ${ }^{4}$ Our patient, when in a deep coma, had increased DWI and reduced ADC signals, the equivalent of cytotoxic edema in acute-phase HE. In addition, MR imaging on the 54th hospital day showed high-intensity areas on the T1-weighted images, indicative of the laminar necrosis, which marks the chronic phase of acute HE. The pathologic process of acute HE was clearly observable in our patient's brain MR imaging series.

Most patients with acute HE do not show abnormal DWI signals. Clinical courses for the reported patients ${ }^{1,2}$ who had DWI abnormalities were very severe and fulminant. We speculate that this is the reason for abnormal image findings in those reports. Accumulation of similar cases is needed to clarify this condition.

$\mathrm{PBC}$ was diagnosed because our patient had no history of alcohol dependence, no viral markers, no elevation of liver enzymes except alkaline phosphatase, and only a positive antimitochondrial $\mathrm{M}_{2}$ antibody specific for PBC. Portal hypertension typically develops during the late stage of chronic liver disease, but $\mathrm{PBC}$ is the exception in some patients. A patient with $\mathrm{PBC}$ may have an accompanying portal systemic shunt in the early histologic stage. ${ }^{5}$ The possibility of shunt encephalopathy should always be considered for a patient with a disturbance of consciousness for which the cause is unclear, even when there is no apparent liver disease.

We have reported a rare case of shunt encephalopathy caused by $\mathrm{PBC}$, in which widespread cortical lesions were present on DWI.

\section{References}

1. Arnold SM, Els T, Spreer J, et al. Acute hepatic encephalopathy with diffuse cortical lesions. Neuroradiology 2001;43:551-54

2. Babington JR, Stahl JH, Coy DL. Reversible cytotoxic edema in a cirrhotic patient following TIPS. J Neuroimaging 2009;19:391-93

3. Sherlock S, Dooley J. Hepatic encephalopathy. In: Disease of the Liver and Biliary System. 9th ed. Oxford, UK; Blackwell Scientific Publications; 1993:86-101

4. Brusilow SW, Horwich AL. Urea cycle enzyme. In: Scriver CR, Beaudete AL, Sly WS, et al, eds. The Metabolic and Molecular Bases of Inherited Disease. 8th ed. New York: McGraw-Hill; 2001:22:843-47

5. Kaplan MM. Primary biliary cirrhosis. In: Schiff L, Schiff ER, eds, Diseases of the Liver. Vol 1. 7th ed. Philadelphia: JB Lippincott; 1993:377-410

S. Toru

K. Matumura

R. Kawaguchi

T. Kobayashi

Department of Neurology

T. Irie

Department of Internal Medicine Nakano General Hospital

Toyko, Japan

DOI 10.3174/ajnr.A2466 